\title{
EXPANSION THEORY ASSOCIATED WITH LINEAR DIFFERENTIAL EQUATIONS AND THEIR REGULAR SINGULAR POINTS*
}

\author{
BY

\section{LEONARD BRISTOW}

Introduction. Birkhoff $\dagger$ has developed an expansion theory associated with linear differential equations on an interval of the real axis containing no singular points of the equation. The purpose of this paper is to develop a similar theory for the domain of complex variables in a region containing a regular singular point of the equation. To take the place of the two-point boundary conditions of the real variable case, we have corresponding relations between the values of the solution and its derivatives at a given point, and the values obtained by analytic continuation about the regular singular point back to the given point. The boundary conditions, $n$ in number, are assumed to be linear, homogeneous and linearly independent, the order of the equation being $n$.

An expansion theory associated with the linear equation of the second order with polynomial coefficients has been developed by O. Volk. $\ddagger$ The method used in this paper is different from that employed by Volk and the coefficients are not assumed to be polynomials. The results of this paper and those of Volk overlap each other for the second-order equation, but neither includes the whole of the other. The Neumann-Gegenbauer expansions in Bessel's functions are obtained as special cases. The Legendre and hypergeometric differential equations give rise to expansion theories if infinity is considered as the regular singular point and the parameter is suitably chosen as a function of the arbitrary constants of the respective equations. The expansion theory for the hypergeometric equation as developed by Reinsch $\S$ is not included as a special case, since the parameter does not enter in the same way. In another paper it is hoped to extend the present theory so as to include the latter as a special case of a more general theory. The Laurent expansion is included as a special case arising from differential equations of every order.

A solution of a differential equation with a regular singular point is, in

* Presented to the Society, April 4, 1931; received by the editors October 30, 1930.

† These Transactions, vol. 9 (1908), p. 373.

‡ Mathematische Annalen, vol. 86 (1922), p. 296.

$\S$ American Journal of Mathematics, vol. 47 (1925), p. 45. 
general, multiple-valued in the neighborhood of the singular point. An infinite series of such solutions in which the type of multiple-valuedness changes from term to term of the series will in general not converge to a function with a simple type of multiple-valuedness. For this and other reasons the boundary conditions will be limited as set forth later.

The method of the paper is to obtain an expansion of $(t-x)^{-1}$ by means of the solutions of the original equation and its adjoint. The expansion of an arbitrary analytic function of one variable is then obtained by use of the Cauchy integral theorem. Absolute and uniform convergence of the expansion is proved by obtaining asymptotic properties of the functions involved. The results are generalized by developing an expansion theory for functions of any finite number of complex variables.

1. Boundary conditions. There is no generality lost by assuming that the regular singular point is at $x=0$. In order that the formulas be as simple as possible we consider the equation $\dagger$ to be

(1) $L(y)+\lambda x^{-1} y \equiv x^{n-1} y^{(n)}+x^{n-2} \alpha y^{(n-1)}$

$$
+x^{n-3} p_{2}(x) y^{(n-2)}+\cdots+p_{n}(x) x^{-1} y+\lambda x^{-1} y=0,
$$

where $p_{2}(x), \cdots, p_{n}(x)$ are analytic and single-valued within a circle of radius $\Gamma$ about $x=0$ and $\alpha$ is a constant. The equation adjoint to (1) may be written

(2) $M(v)+\lambda x^{-1} v \equiv(-1)^{n} x^{n-1} v^{(n)}+x^{n-2} \beta v^{(n-1)}$

$$
+x^{n-3} q_{2}(x) v^{(n-2)}+\cdots+q_{n}(x) x^{-1} v+\lambda x^{-1} v=0,
$$

where $q_{2}(x), \cdots, q_{n}(x), \beta\left(q_{n}(x) \equiv p_{n}(x)\right)$ have the same properties as $p_{2}(x)$, $\cdots, p_{n}(x), \alpha$. We have the Lagrange identity

$$
v L(y)-y M(v) \equiv \frac{d}{d x} R(y, v),
$$

where $R(y, v)$ is the bilinear concomitant. Let

$$
\begin{aligned}
-F(\rho)= & \rho(\rho-1) \cdots(\rho-n+1)+\alpha \rho(\rho-1) \cdots(\rho-n+2) \\
& +\sum_{\mu=2}^{n} p_{\mu}(0) \rho(\rho-1) \cdots(\rho-n+\mu+1) .
\end{aligned}
$$

$\dagger$ The equation

$$
z^{n-1} u^{(n)}+z^{n-2} p_{1}(z) u^{(n-1)}+\cdots+p_{n}(z) z^{-1} u+R(z) \lambda z^{-1} u=0
$$

is not essentially more general if $R(0) \neq 0$, since substituting

$$
u=\exp \left[-\frac{1}{n} \int_{a}^{z} \frac{p_{1}(t)-p_{1}(0)}{t} d t\right], \quad z=\exp \left[\int_{b}^{x}\{R(t)\}^{1 / n} t^{-1} d t\right],
$$

$a \neq 0, b \neq 0$, in the order given transforms it into the type (1). 
Then the indicial equation of (1) for $x=0$ is

$$
\lambda-F(\rho)=0 .
$$

If $\rho_{1}, \rho_{2}, \cdots, \rho_{n}$ are the exponents belonging to the point $x=0$ of the original equation (1), then, since we have taken the equation in this form, $-\rho_{1},-\rho_{2}, \cdots,-\rho_{n}$ are the corresponding exponents for (2). This fact may be easily inferred from a result in Forsyth's Theory of Differential Equations, Part III, volume IV, pages 256-257.

Let $y^{(j)}(a)$ be the value at $x=a$ of the $j$ th derivative of $y(x)$, a solution of the differential equation, and let $Y^{(j)}(a)$ be the value obtained for $y^{(j)}(x)$ at $x=a$ after analytic continuation about $x=0$. The $2 n$ quantities $y^{(j)}(a)$, $Y^{(j)}(a), j=0,1, \cdots, n-1$, are the quantities used in the boundary conditions. We assume a set of boundary conditions which have, in addition to the properties described in the introduction, the following properties: they determine an enumerable infinitude of values of $\lambda$, called characteristic values, for each of which the equation has a solution (not identically zero) satisfying the boundary conditions; for each characteristic value the solution for the present is assumed unique. The case where the solution is not unique is discussed separately for the boundary conditions which are used. The boundary conditions to be associated with the adjoint equation are to have the same properties. We shall later put on still further restrictions and arrive at the boundary conditions

$$
Y^{(j)}(a)=K y^{(j)}(a) \quad(j=0,1, \cdots, n-1),
$$

for the original equation; for the adjoint equations we shall then have the conditions

$$
V^{(j)}(a)=K^{-1} v^{(j)}(a) \quad(j=0,1, \cdots, n-1) .
$$

Let $\lambda^{*}$ and $\lambda^{* *}$ be two different characteristic values for a given set of boundary conditions, and let $y^{*}(x), y^{* *}(x)$ be solutions of (1) for $\lambda=\lambda^{*}$ and $\lambda=\lambda^{* *}$ respectively, and let $v^{*}(x)$ and $v^{* *}(x)$ be the corresponding solutions of the adjoint equation. Suppose $\rho_{1}, \rho_{2}, \cdots, \rho_{n}$ to be the exponents corresponding to $\lambda=\lambda^{* *}$ and $\sigma_{1}, \sigma_{2}, \cdots, \sigma_{n}$ be those corresponding to $\lambda=\lambda^{*}$. Since a differential equation having two exponents equal or differing by an integer usually has in its general solution logarithms, we shall assume, except for a finite number at most of characteristic values, that the exponents are not equal and no two differ by an integer. Consider the $n^{2}$ differences $\rho_{i}-\sigma_{j}$, $i, j=1,2, \cdots, n$. We shall make the additional assumption that not more than one of these differences is equal to another difference plus an integer. The reason for this will appear later. 
The solutions in question for the original equation and its adjoint $\lambda=\lambda^{*}$ may be written

$$
\begin{aligned}
& y^{*}(x)=c_{1} y_{\sigma_{1}}(x)+\cdots+c_{n} y_{\sigma_{n}}(x), \\
& v^{*}(x)=b_{1} v_{-\sigma_{1}}(x)+\cdots+b_{n} v_{-\sigma_{n}}(x),
\end{aligned}
$$

where the functions in the right members have the formulas

$$
\begin{aligned}
& y_{\sigma_{i}}(x)=x^{\sigma_{i}}\left\{1+\sum_{m=1}^{\infty} a_{\sigma_{i}, m} x^{m}\right\}=x^{\sigma_{i}} \phi_{\sigma_{i}}(x), \\
& v_{-\sigma_{i}}(x)=x^{-\sigma_{i}}\left\{1+\sum_{m=1}^{\infty} b_{-\sigma_{i}, m} x^{m}\right\}=x^{-\sigma_{i} \psi_{-\sigma i}}(x),
\end{aligned}
$$

and similarly for $\lambda=\lambda^{* *}$. The series (7) and (8) converge for $|x|<\Gamma$.

We have from (3)

$$
\begin{aligned}
v^{*} L\left(y^{* *}\right)-y^{* *} M\left(v^{*}\right)+\left(\lambda^{* *}-\lambda^{*}\right) v^{*} y^{* *} x^{-1} & \\
& \equiv \frac{d}{d x}\left\{R\left(y^{* *}, v^{*}\right)\right\}+\left(\lambda^{* *}-\lambda^{*}\right) v^{*} y^{* *} x^{-1}=0 .
\end{aligned}
$$

Multiply the above by $d x$ and integrate once about the origin along the contour $C_{a}$ which passes through the point $x=a$ and encloses only the singularity $x=0$ of the equation. The first part becomes $R\left(y^{* *}(a), v^{*}(a)\right)-R\left(Y^{* *}(a)\right.$, $\left.V^{*}(a)\right)$. This difference is a non-singular $\dagger$ bilinear form in the $2 n$ quantities involved. Hence if the original conditions were linear, homogeneous, linearly independent, and $n$ in number there exist unique boundary conditions for the adjoint such that if $v^{*}$ is a solution satisfying these conditions the foregoing expression has the value zero. Letting $v^{*}$ be such a solution, we have, since $\lambda^{*} \neq \lambda^{* *}$

$$
\int_{C_{a}} v^{*} y^{* *} x^{-1} d x=0
$$

This condition together with the condition

$$
\int_{C_{a}} v^{*} y^{*} x^{-1} d x \neq 0
$$

would enable us to determine formally the coefficients in the expansion

$$
f(x)=\sum_{m=0}^{\infty} a_{m} y_{m}(x),
$$

† This follows from the formal properties of the bilinear concomitant as in the real variable case. Compare Carmichael, American Journal of Mathematics, vol. 43 (1921), p. 234. 
where $y_{m}(x)$ is the solution, supposed for the present to be unique, satisfying the original equation and boundary conditions for $\lambda=\lambda_{m}$, a characteristic value. However, in order to do this using only the foregoing, the path of integration would have to pass through the point $x=a$. We would then be unable to obtain the expansions of functions whose region of existence did not extend out to $x=a$. We shall then limit ourselves to boundary conditions for which (9) is true for all paths which enclose only the singularity $x=0$.

At any point sufficiently near $x=0$ the function $v^{*} y^{* *} x^{-1}$ is single-valued and analytic. There exists then a function $\psi(x)$ such that its derivative is $v^{*} y^{* *} x^{-1}$. If we continue analytically about $x=0$ the analytic continuation of $\psi(x)$ retains this property. From (9) for all paths we conclude that $\psi(x)$ is single-valued near $x=0$. Its derivative, and hence $v^{*} y^{* *}$, has this property.

We have the following $\dagger$

Lemma. If $S_{1}(x), S_{2}(x), \cdots, S_{k}(x), T(x)$ are single-valued and analytic functions near $x=0$ and $\alpha_{1}, \cdots, \alpha_{k}$ are complex numbers different from integers and such that no difference $\alpha_{i}-\alpha_{j}, i, j=1,2, \cdots, k ; i \neq j$, is an integer, and we have

$$
x^{\alpha_{1}} S_{1}(x)+x^{\alpha_{2}} S_{2}(x)+\cdots+x^{\alpha_{k}} S_{k}(x) \equiv T(x),
$$

it follows that $S_{1}(x) \equiv S_{2}(x) \equiv \cdots \equiv S_{k}(x) \equiv T(x) \equiv 0$.

For the conditions named we shall show that the boundary conditions must lead to solutions which are constant multiples of the fundamental solutions corresponding to one exponent for each characteristic value. Suppose on the contrary that $y^{* *}$ and $v^{*}$ are both combinations of more than one solution corresponding to the exponents. The product $y^{* *} v^{*}$ is of the form of the left member of the equation of the lemma with the ' $\alpha$ 's replaced by $\rho_{s}-\sigma_{p}$. One of the differences must be an integer. If this were not true the product would be identically zero by the lemma, and hence either $v^{*}$ or $y^{* *}$ would be identically zero, contrary to hypothesis. We may then take $\rho_{1}-\sigma_{1}=m_{1}, m_{1}$ an integer and $c_{1} \neq 0$ and $b_{1} \neq 0$. If this were the only such difference equal to an integer, then by the lemma the product would be equal to $c_{1} b_{1} x^{p_{1}-\sigma_{1}} \phi_{p_{1}}(x)$ $\cdot \psi_{-\sigma_{1}}(x)$. We would then have

$$
b_{1} c_{2} x^{\rho_{2}-\sigma_{1}} \phi_{\rho_{2}}(x) \psi_{-\sigma_{1}}(x)+b_{2} c_{1} x^{\rho_{1}-\sigma_{2}} \phi_{\rho_{1}}(x) \psi_{-\sigma_{2}}(x)+\cdots+\cdots \equiv 0 .
$$

Now $\phi_{\rho_{i}}(x) \not \equiv 0$ and $\psi_{-\sigma_{i}}(x) \not \equiv 0, i=1,2, \cdots, n$. We shall first suppose that the notation can be so chosen that $c_{2} \neq 0, b_{2} \neq 0$. Since the above equation is satisfied and none of the members written is identically zero, the lemma is

$\dagger$ For proof see for instance Schlesinger, Handbuch der Theorie der linearen Differentialgleichungen, vol. I, p. 176. 
contradicted unless the differences $\rho_{1}-\sigma_{2}, \rho_{2}-\sigma_{1}, \rho_{2}-\sigma_{2}$ are equal to similar differences plus an integer. We have at least two such and this is contrary to hypothesis. Hence at least either $v^{*}$ or $y^{* *}$ is of the character we wished to establish for them. Next suppose $c_{2}=c_{3}=\cdots=c_{n}=0$ but $b_{2} \neq 0$. Then we would have to have either $\rho_{1}-\sigma_{2}=m_{2}$ or $\rho_{1}-\sigma_{2}=\rho_{1}-\sigma_{j}+m_{3}, j>2, m_{2}$ and $m_{3}$ being integers. In both cases using the relation $\rho_{1}-\sigma_{1}=m_{1}$ we would have $\sigma_{i}-\sigma_{j}=$ integer, $i \neq j$, contrary to hypothesis. Hence $b_{2}=b_{3}=\cdots=b_{n}=0$. Similarly if we chose $c_{2} \neq 0$ and $b_{2}=b_{3}=\cdots=b_{n}=0$ we would get a contradiction. The same result would be obtained if it were assumed that another difference of exponents were an integer.

If $\nu$ is the exponent to which the solution satisfying the boundary conditions corresponds for $\lambda=\lambda_{0}$, then the exponents for the other characteristic values are $\nu+m, m$ a positive or negative integer. From (5) we have

$$
\lambda_{m}=F(\nu+m) \quad(m=0, \pm 1, \pm 2, \cdots) .
$$

The solutions of the original equation evidently satisfy the boundary conditions

$$
\begin{aligned}
Y(a) & =K y(a), \\
Y^{(s)}(a) & =K y^{(s)}(a) \quad(s=1, \cdots, n-1) ;
\end{aligned}
$$

and the adjoint solutions satisfy the conditions

$$
\begin{aligned}
V(a) & =K^{-1} v(a), \\
V^{(s)}(a) & =K^{-1} v^{(s)}(a) \quad(s=1, \cdots, n-1),
\end{aligned}
$$

where $K=e^{2 \pi i v}, i=(-1)^{1 / 2}$ and $a$ is any point different from zero and within the circle of radius $\Gamma$ about $x=0$. The adjoint conditions have the required character that the bilinear concomitant is single-valued near $x=0$. Any other set having this character is made up of linear combinations of these and hence equivalent.

These boundary conditions can be seen to lead to the characteristic values $\lambda_{m}$ and to the solutions we have obtained. We have the orthogonality conditions

$$
\int_{C} v_{-\nu-s}(x) y_{\nu+m}(x) x^{-1} d x=0, s \neq m
$$

where $C$ is a circle of radius $R<\Gamma$ about $x=0$. The arbitrary multipliers may be taken equal to unity since they can be absorbed in the coefficients of the expansion. It is evident from (7) and (8) that

$$
\int_{C} v_{-\nu-m}(x) y_{\nu+m}(x) x^{-1} d x=2 \pi i .
$$


The set $\lambda_{m}=F(\nu+m)$ will furnish an enumerably infinite number of characteristic values as required for the expansion. Unless $F(\nu+m)=F(\nu+p)$, $m \neq p$, the equation has only one solution satisfying the boundary conditions. The case $F(\nu+m)=F(\nu+p)$ we shall treat separately.

2. Formal expansion of $x^{v} /(t-x)$. For a given value of $K \neq 0$ of the boundary conditions we have for some $\nu, e^{2 \pi i v}=K$. Let $\nu$ be a determination such that either (a) a solution of the form (7) exists $\dagger$ for each exponent $\nu+m, m=0,1,2, \cdots$, even if $F(\nu+m)=F(\nu+p), m \neq p$, or (b) the members of the set $F(\nu+m), m=0,1,2, \cdots$, are all different. We shall see presently that we can do this. The set of characteristic values of $\lambda$ are $\lambda_{m}=F(\nu+m)$ for $m$ a positive or negative integer or zero. For our expansion theory we will need only those arising from $m \geqq 0$.

If for a given characteristic value $\lambda_{m}$ the exponents are such that two of them differ by an integer or are equal but the exponent $\nu+m$ is not involved, the orthogonality conditions still hold. However, if the two exponents whose difference is an integer include as one of them the exponent $\nu+m$ we would have

$$
\lambda_{m}=F(\nu+m)=F(\nu+p)=\lambda_{p}, p \neq m .
$$

That is, there are two notations for the same characteristic value. If $p$ is negative there would be the question as to the existence of the solution of the adjoint corresponding to the exponent $-\nu-m$, since $-\nu-p$ is also an exponent. If this solution exists in the form (8) there is no change in the theory.

For a given $\nu$ we have $\lambda_{m}=F(\nu+m)=G(m)$, where

$$
\begin{aligned}
G(m) & =-m^{n}+a_{1} m^{n-1}+\cdots+a_{n} \\
& =-m^{n}+\alpha_{1} m^{n-1}+\cdots+\alpha_{n}+i\left\{\beta_{1} m^{n-1}+\cdots+\beta_{n}\right\},
\end{aligned}
$$

$a_{s}=\alpha_{s}+i \beta_{s}, \alpha_{s}$ and $\beta_{s}$ being real. Then $F(\nu+m)=F_{1}(m)+i F_{2}(m)$ where $F_{1}(m)$ and $F_{2}(m)$ are real-valued polynomials in the real variable $m$. Now $F_{1}(m)$ and $F_{2}(m)$ as functions of the continuous variable $m$ have a finite number of maxima and minima, and hence for $m$ sufficiently large $F_{1}(m)$ is different for different values $m$, and likewise for $F_{2}(m)$. From this it follows that at most only a finite number of $F(\nu+m), m=0,1, \cdots$, are equal.

We shall now compute formally the coefficients in the expansion

$$
x^{\nu} /(t-x) \sim \sum_{m=0}^{\infty} a_{m}(t) y_{v+m}(x) .
$$

$\dagger$ For the condition that this solution exists see Ince, Ordinary Differential Equations, Chapter XVI, pp. 404-6. 
We shall first consider the case when the $\lambda$ 's with the positive subscripts are all distinct from those with negative subscripts. Let $\lambda_{m}$ be a characteristic value which does not have two exponents $\nu+m$ and $\nu+p, p \neq m$, corresponding to it. Multiply both sides of (15) through by $v_{-\nu-m}(x) x^{-1} d x$ and assume for the moment that the series on the right permits of term by term integration. Integrating on the contour $C$ about the origin and using (13) and (14) we get

$$
a_{m}(t)=\frac{1}{2 \pi i} \int_{C} \frac{x^{\nu} v_{-v-m}(x) d x}{x(t-x)} .
$$

Substituting series (8) for $v_{-r-m}(x)$ and expanding $1 /(t-x)$ in powers of $x$ and simplifying we get

$$
a_{m}(t)=t^{-1} P_{-\nu-m}(t),
$$

where $P_{-,-m}(t)$ is the sum of the first $m+1$ terms of the power series expansion of $t^{n} v_{->-m}(t)$.

Next suppose $F\left(\nu+r_{1}\right)=F\left(\nu+r_{2}\right)=\cdots=F\left(\nu+r_{s}\right)$, where $0 \leqq r_{1}<r_{2}<$ $\cdots<r_{s}$, and that solutions exist in the form (7) for the original equation and adjoint for the exponents $\nu+r_{1}, \nu+r_{2}, \cdots, \nu+r_{\text {s }}$ and $-\nu-r_{1},-\nu-r_{2}$, $\cdots,-\nu-r_{\text {o }}$ respectively. Let $u_{\nu+r_{1}}(x), \cdots, u_{\nu+r_{0}}(x)$ be a particular set of solutions of the original equation and $v_{-v-r_{1}}(x), \cdots, v_{-n-r_{4}}$ be a particular set for the adjoint. These sets may be chosen, for example, by putting all the arbitrary elements equal to unity for each exponent. We have

$$
\begin{aligned}
\int_{c} v_{-v-r_{q}}(x) u_{v+r_{q}}(x) x^{-1} d x & =0, q<p, \\
& =2 \pi i, q=p, \\
& =d_{q, p} \cdot 2 \pi i, q>p,
\end{aligned}
$$

where $d_{q, p}$ is not necessarily zero. Now the solution

$$
y_{v+r p}(x)=u_{v+r_{p}}(x)+\alpha_{p, p+1} u_{v+r_{p+1}}(x)+\cdots+\alpha_{p, s} u_{v+r_{s}}(x)
$$

is in the form of (7). Next form

$$
\int_{C} v_{-\boldsymbol{\nu}-r_{q}}(x) y_{v+r_{p}}(x) x^{-1} d x=l_{q, p} 2 \pi i,
$$

for $q=p+1, \cdots, s$. We then have $s-p$ equations

$$
\begin{aligned}
d_{p+1, p}+\alpha_{p, p+1} & =l_{p+1, p}, \\
d_{p+2, p}+\alpha_{p, p+1} d_{p+2, p+1}+\alpha_{p, p+2} & =l_{p+2, p}, \\
\cdots \cdots \cdots \cdots & \cdots \cdots \\
d_{s, p}+\alpha_{p, p+1} d_{s, p+1}+\cdots+\alpha_{p, s} & =l_{s, p} .
\end{aligned}
$$


It is evident that we can determine uniquely $\alpha_{p, q}, q=p+1, \cdots, s$, so that $l_{q, p}=0, q=p+1, \cdots, s$. Let this be done for $p=1,2, \cdots, s$. We then have

$$
\begin{aligned}
\int_{C} v_{-\nu-r_{q}}(x) y_{\nu+r_{p}}(x) x^{-1} d x & =0, q \neq p, \\
& =2 \pi i, q=p,
\end{aligned}
$$

and the solutions have the same orthogonality properties as do the remaining solutions if this is done for every such case. The determination of the coefficients in the expansion is then the same as before. We have the formal expansion

$$
x^{v} /(t-x) \sim \sum_{m=0}^{\infty} t^{-1} P_{-v-m}(t) y_{v+m}(x) .
$$

We now take up the case when we have, for some $\lambda_{m}, \lambda_{m}=\lambda_{-p}, m$ and $p$ both positive. If a solution of the adjoint exists for the exponent $-\nu-m$ there is no change in the theory. However even if this solution does not exist we sometimes can obtain a formal expansion. In $P_{-\nu-m}(t)$ we have only the first $(m+1)$ terms of the power series expansion of $t^{p} v_{-\nu-m}(t)$. If we try by formal methods to find the solution of the adjoint corresponding to the exponent $-\nu-m$ we are able to compute the coefficients up to and including $b_{-v-m, m+p-1}$, being unable to compute $b_{-v-m, m+p}$ on account of a zero multiplier. Since $p>0$ we can compute the coefficients up to $b_{-r-m, m}$ which are all that are used in $P_{-v-m}(t)$. Whenever this case arises we shall use the coefficients for $P_{-\nu-m}(t)$ computed in this way. It is to be noted that these coefficients satisfy the same general formulas as do those when the solution exists.

3. Asymptotic properties of $y_{p+m}(x)$ and $P_{-v-m}(t)$ with respect to $m$. We now show that we have an actual expansion of $x^{\nu} /(t-x)$ by first showing that the series in (17) may be integrated term by term and next that it converges to $x^{\prime \prime} /(t-x)$. This will be done by first proving the uniform convergence of the series in (17) with respect to both $x$ and $t$ in a suitable range.

The functions $y_{v+m}(x)$ and $P_{-v-m}(t)$ of (17) may be written

We have $†$

$$
\begin{aligned}
& y_{v+m}(x)=\sum_{s=0}^{\infty} a_{\nu+m, s} x^{s+\nu}, a_{\nu+m, 0}=1, \\
& P_{-\nu-m}(t)=\sum_{s=0}^{m} b_{-\nu-m, t^{t}}, b_{-\nu-m, 0}=1 .
\end{aligned}
$$

† For formulas (19) to (24) see Frobenius, Journal für Mathematik, vol. 76 (1873), p. 214. 


$$
\begin{aligned}
a_{\nu+m, 0} f_{0}(\nu+m) & =0, \\
a_{\nu+m, 1} f_{0}(\nu+m+1)+a_{\nu+m, 0} f_{1}(\nu+m) & =0
\end{aligned}
$$

$$
a_{\nu+m, s+1} f_{0}(\nu+m+s+1)+\cdots+a_{\nu+m, 0} f_{s+1}(\nu+m)=0,
$$

and

$$
\begin{aligned}
& b_{-\nu-m, 0} \phi_{0}(-\nu-m)=0, \\
& b_{\rightarrow-m, 1} \phi_{0}(-\nu-m+1)+b_{->-m, 0} \phi_{1}(-\nu-m)=0 \text {, } \\
& b_{-\nu-m, m} \phi_{0}(-\nu)+\cdots+b_{-\nu-m, 0} \phi_{m}(-\nu-m)=0 \text {, }
\end{aligned}
$$

where

$f(x, \nu+m+s)=[\nu+m+s]_{n}+\alpha[\nu+m+s]_{n-1}+p_{2}(x)[\nu+m+s]_{n-2}$

$$
\begin{aligned}
& +\cdots+p_{n}(x)+\lambda_{m} \\
= & \sum_{\mu=0}^{\infty} f_{\mu}(\nu+m+s) x^{\mu},
\end{aligned}
$$

$$
\begin{aligned}
\phi(x,-\nu-m+s)= & (-1)^{n}[-\nu-m+s]_{n}+\beta[-\nu-m+s]_{n-1} \\
& +q_{2}(x)[-\nu-m+s]_{n-2}+\cdots+q_{n}(x)+\lambda_{m} \\
= & \sum_{\mu=0}^{\infty} \phi_{\mu}(-\nu-m+s) x^{\mu}
\end{aligned}
$$

$[\nu+m+s]_{r}=(\nu+m+s)(\nu+m+s-1) \cdots(\nu+m+s-r+1)$.

The first conditions in (19) and (20) are

$$
f_{0}(\nu+m)=\lambda_{m}-F(\nu+m)=0 \text { and } \phi_{0}(-\nu-m)=\lambda_{m}-F(\nu+m)=0 ;
$$

both give rise to the indicial equation. If

(22) $C_{n+m, s+1}$

$=\frac{\left\{\left|a_{\nu+m, 0}\right| M(\nu+m+s)+\left|a_{\nu+m, s-1}\right| M(\nu+m+s-1) R^{-1}+\cdots+M(\nu+m) R^{-\bullet}\right\}}{\left|f_{0}(\nu+m+s+1)\right|}$,

where

$$
M(\nu+m+s)=\left|[\nu+m+s]_{n-2}\right| M_{2}+\cdots+M_{n},
$$

$M_{2}, \cdots, M_{n}$ being the maxima of $\left|p_{2}^{\prime}(x)\right|$, etc., within and on a circle of radius $R<\Gamma$, then

$$
\left|a_{v+m, s+1}\right|<C_{v+m, s+1} \text {. }
$$


We also have

$$
C_{\nu+m, s+1}<C_{\nu+m, s}\left\{\frac{M(\nu+m+s)+\left|f_{0}(\nu+m+s)\right| R^{-1}}{\left|f_{0}(\nu+m+s+1)\right|}\right\},
$$

which becomes

$$
C_{\nu+m, s+1}<C_{\nu+m, s}\left\{\frac{M(\nu+m+s)+|F(\nu+m)-F(\nu+m+s)| R^{-1}}{|F(\nu+m)-F(\nu+m+s+1)|}\right\} .
$$

For $m$ sufficiently large $\nmid$ the denominator of the right-hand side of (24) is always different from zero. We consider first

$$
P_{\nu+m, s}=\frac{F(\nu+m)-F(\nu+m+s)}{F(\nu+m)-F(\nu+m+s+1)}=\frac{G(m+s)-G(m)}{G(m+s+1)-G(m)}
$$

when $s$ is large. Put

$$
T_{m+s}(r)=\frac{(m+s)^{n-r}}{(m+s)^{n}-m^{n}}, \quad U_{m+s}(r)=\frac{m^{n-r}}{(m+s)^{n}-m^{n}} .
$$

After dividing the numerator and denominator of (24) by $(m+s)^{n}-m^{n}$ and expanding $(m+s+1)^{n-r}$ into powers of $m+s$ by the binomial theorem, we may write

$$
P_{-r-m, s}=\frac{1+\sum_{r=1}^{n}\left\{B_{r} T_{m+s}(r)+C_{r} U_{m+\varepsilon}(r)\right\}}{1+\sum_{r=1}^{n}\left\{D_{r} T_{m+s}(r)+E_{r} U_{m+s}(r)\right\}},
$$

where $B_{r}, C_{r}, D_{r}$, and $E_{r}$ are uniquely determined. Now

and $m /(m+s)<1 ;$ hence

$$
\frac{(m+s)^{n-r}}{(m+s)^{n}-m^{n}}=\frac{1}{(m+s)^{r}-m^{r}\left\{\frac{m}{m+s}\right\}^{n-r}},
$$

Now we have

$$
\frac{(m+s)^{n-r}}{(m+s)^{n}-m^{n}}<\frac{1}{(m+s)^{r}-m^{r}} .
$$

$$
(m+s)^{r}-m^{r}=s\left\{r m^{r-1}+\frac{r(r-1)}{2} m^{r-2} s+\cdots+s^{r-1}\right\} \geqq s, r \geqq 1 ;
$$

$\dagger$ Note that the argument for $y_{v+m}(x)$ holds for $m$ becoming infinite through positive real values. 
hence

$$
\frac{(m i+s)^{n-r}}{(m+s)^{n}-m^{n}}<\frac{1}{s}, \frac{m^{n-r}}{(m+s)^{n}-m^{n}}<\frac{(m+s)^{n-r}}{(m+s)^{n}-m^{n}}<\frac{1}{s} .
$$

If $s$ is sufficiently large the denominator is surely positive and we can write

$$
\left|P_{r+m, s}\right|<\frac{1+\sum_{r=1}^{n}\left\{\left|B_{r}\right| T_{m+s}(r)+\left|C_{r}\right| U_{m+s}(r)\right\}}{1-\sum_{r=1}^{n}\left\{\left|D_{r}\right| T_{m+s}(r)+\left|E_{r}\right| U_{m+s}(r)\right\}} .
$$

For a given $\delta, 1>\delta>0$, there exists an $s_{1}$ such that for $s>s_{1}$

$$
\left|P_{v+m, s}\right|<\frac{1+\delta}{1-\delta}
$$

Now consider one term of

$$
\begin{aligned}
& \frac{M(\nu+m+s)}{|F(\nu+m)-F(\nu+m+s+1)|} \\
& =\sum_{r=2}^{n} \frac{M_{r}|(\nu+m+s) \cdots(\nu+m+s-n+r-1)|}{|G(m+s+1)-G(m)|}
\end{aligned}
$$

which may be written

$$
\begin{aligned}
Q_{r+m, \text { o }} & =\frac{M_{r}|(\nu+m+s) \cdots(\nu+m+s-n+r-1)|}{|G(m+s+1)-G(m)|} \\
& =\frac{M_{r}\left|\left(1+\frac{\nu}{m+s}\right) \cdots\left(1+\frac{\nu-n+r-1}{m+s}\right)\right|}{\left\{(m+s)^{r}-\left(\frac{m}{m-s}\right)^{n-r} m^{r}\right\} \frac{|G(m+s+1)-G(m)|}{(m+s)^{n}-m^{n}}} .
\end{aligned}
$$

We have for $s>s_{1}$

$$
Q_{v+m, s}<\frac{\bar{M}_{r}}{(m+s)^{r}-m^{r}}<\frac{\bar{M}_{r}}{s(1-\delta)},
$$

where $\bar{M}_{r}$ is independent of $s$. For a given $\delta$ there exists an $s_{2}$ such that for $s>s_{2} \geqq s_{1}$

$$
Q_{r+m, 2}(r)<\frac{\delta}{n-1} \quad(r=2,3, \cdots, n) .
$$

We can now write 


$$
C_{\nu+m, s+1}<C_{\nu+m, 8}\left\{\delta+\frac{1+\delta}{1-\delta} R^{-1}\right\}<\frac{C_{p+m, s}}{R-\delta_{2}}, s>s_{2} \geqq s_{1},
$$

where $\delta_{2}$ may be made arbitrarily small.

For $s=1,2, \cdots, s_{2}$ the right-hand side of (24) approaches $s /((s+1) R)$ $<1 / R$ as $m$ becomes infinite. This follows from the fact that the degree of the numerator and of the denominator of $P_{v+m, e}$ is $n-1$ in $m$, and the coefficients of $m^{n-1}$ are respectively $s$ and $s+1$. The rest of the numerator of the right-hand side of (24) is of degree $n-2$ in $m$. There exists then an $m_{1}$ such that for all $m>m_{1}$ we have for all $s$

$$
C_{v+m, 0+1}<\frac{C_{n+m, \bullet}}{R-\delta_{2}}
$$

Using this bound for $C_{p+m, 8}, \cdots, C_{p+m, 2}$ we get

where

$$
\left|a_{v+m, s+1}\right|<C_{p+m, s+1}<\left[\frac{1}{R-\delta_{2}}\right]^{\bullet} C_{p+m, 1}
$$

$$
C_{\nu+m, 1}=\frac{M_{2}\left|[\nu+m]_{n-2}\right|+\cdots+M_{n}}{|F(\nu+m)-F(\nu+m+1)|} .
$$

We may write

$$
\begin{aligned}
\left|x^{-\nu-m} y_{\nu+m}(x)-1\right| & <C_{\nu+m, 1} \sum_{\delta=1}^{\infty}\left[\frac{1}{R-\delta_{2}}\right]^{s-1}|x|^{\cdot} \\
& =C_{\nu+m, 1}|x|\left[\frac{1}{1-\frac{|x|}{R-\delta_{2}}}\right],|x|<R-\delta_{2} .
\end{aligned}
$$

Now for $|x| \leqq R^{\prime}<R-\delta_{2}<\Gamma$ we have for $M$ independent of $x$ and $m$.

$$
\left|x^{-\nu-m} y_{v+m}(x)-1\right|<C_{\nu+m, 1} M \text {. }
$$

The numerator of $C_{v+m, 1}$ is of degree $n-2$ in $m$ and the denominator of degree $n-1$. Hence for a given arbitrary small positive number $\epsilon$ there exists an $m_{2}$ such that for $m>m_{2}$ we have

$$
C_{v+m, 1}<\frac{\epsilon}{M}
$$

Then for $m>m_{3}$, the larger of $m_{1}$ and $m_{2}$, we have

$$
\left|x^{-\nu-m} y_{\nu+m}(x)-1\right|<\epsilon,|x| \leqq R^{\prime}<R-\delta_{2}<\Gamma,
$$

or 


$$
y_{v+m}(x)=x^{\nu+m}\left\{1+\eta_{\nu+m}(x)\right\},
$$

where

$$
\underset{m \rightarrow \infty}{\mathrm{L}} \eta_{v+m}(x)=0 .
$$

We have proved the following

THEOREM I. Let $y_{v+m}(x)$ be the solution of the differential equation with $\lambda=\lambda_{m}$ formed with the exponent $\nu+m$; then if we write

$$
y_{v+m}(x)=x^{\nu+m}\left\{1+\eta_{v+m}(x)\right\}
$$

we have

$$
\underset{m \rightarrow \infty}{\mathrm{L}} \eta_{p+m}(x)=0
$$

provided that $|x| \leqq R^{\prime}<\Gamma$.

The coefficients in the function $P_{-\nu-m}(t)$ for all the cases we have considered satisfy equation (19). Since $\phi_{0}(-\nu-m+s+1)=\lambda_{m}-F(\nu+m+s-1)$ the argument in this case goes much like the preceding when we observe that $m-s$ is always positive. It is necessary that $m$ is always as large as $s$ is taken. We shall not give the proof but state the corresponding

TheOREM II. Let $P_{-v-m}(t)$ be the function previously defined; then if we write

$$
P_{-\nu-m}(t)=t^{-m}\left\{1+r_{-\nu-m}(t)\right\}
$$

we have

provided that $|t| \leqq R^{\prime \prime}<\Gamma$.

$$
\underset{m \rightarrow \infty}{\mathrm{L}} r_{-\nu-m}(t)=0
$$

The following corollary is evident.

CoRollary. Let $y_{v+m}(x)$ and $P_{-\nu-m}(t)$ be the functions of Theorems I and II; then $x^{-v} y_{v+m}(x)$ and $P_{-v-m}(t)$ are dominated by $M_{1}|x|^{m}$ and $M_{2}|t|^{-m}$ respectively, where $M_{1}$ and $M_{2}$ are independent of $x$ and $t$, provided $|x| \leqq R^{\prime}<\Gamma$ and $|t| \leqq R^{\prime \prime}<\Gamma$.

4. Convergence of the expansion for $x^{\nu} /(t-x)$. Consider the series

$$
\sum_{m=0}^{\infty} P_{-v-m}(t) t^{-1} x^{-v} y_{v+m}(x),
$$

where $P_{-\nu-m}(t)$ and $y_{\nu+m}(x)$ are the functions previously defined. By the 
corollary of the preceding section the general term is in absolute value dominated by $M_{1} M_{2}|x|^{m}|t|^{-m-1}$. This is a term of a convergent series if $|x|<|t|$ $<\Gamma$. Hence for $|x|<|t|$ the series converges absolutely. If $t$ is restrained to the circle $|t|=R^{\prime}$ and $|x| \leqq R^{\prime \prime}<R^{\prime}$, then the series is dominated term by term by a converging series of constants and hence by the Weierstrass $M$ test converges absolutely and uniformly with respect to both $t$ and $x$. Next we must show that the series actually represents $1 /(t-x)$.

We prove the following

LEMma. If $y_{p+m}(x)$ and $P_{-v-p}(x)$ are the functions defined above, then

and

$$
\begin{aligned}
\int_{C} P_{-\nu-p}(x) x^{-p} x^{-1} y_{\nu+m}(x) d x & =2 \pi i, p=m, \\
& =0, \quad p \neq m,
\end{aligned}
$$

$$
\frac{1}{2 \pi i} \int_{C} \frac{P_{-\nu-p}(x) d x}{x(t-x)}=t^{-1} P_{-\nu-p}(t) .
$$

The first and last results may be verified easily by using the expansions of the functions involved. We now prove the second result for the cases where the functions $v_{-v-p}(x)$ exist for each $p \geqq 0$. From the orthogonality conditions we have

$$
\begin{aligned}
\int_{C} v_{-\nu-p}(x) y_{\nu+m}(x) x^{-1} d x=\int_{C} P_{-\nu-p}(x) & x^{-\nu-1} y_{\nu+m}(x) d x \\
& +\int_{C} R_{-\nu-p}(x) x^{-\nu} y_{\nu+m}(x) d x=0,
\end{aligned}
$$

where $R_{-p-p}(x)$ is analytic at $x=0$. But

hence

$$
\int_{C} R_{-v-p}(x) x^{-v} y_{v+m}(x) d x=0 ;
$$

$$
\int_{C} P_{-\nu-p}(x) x^{-\nu-1} y_{v+m}(x) d x=0 .
$$

Evaluating the foregoing integral for $m<p$ we get

$$
\sum_{q=0}^{p-m} b_{-v-p, q} \cdot a_{v+m, p-m-q}=0 .
$$

Now let $P_{-v-p}(x)$ be the function defined when the solution does not exist. We evidently have 


$$
\begin{aligned}
\int_{C} P_{-\nu-p}(x) x^{-\nu-1} y_{\nu+m}(x) d x & =0, m>p, \\
& =2 \pi i \sum_{\varphi=0}^{p-m} b_{-\nu-p, q} a_{\nu+m, p-m-q}, m<p, \\
& =2 \pi i, m=p .
\end{aligned}
$$

Consider now the points $\delta$ for which $F(\nu+\delta+m)=F(\nu+\delta+r)$ for every pair $r$ and $m, r \neq m$. For each pair there are at most $n$. The totality for all such pairs forms an enumerable set. The complementary set, then, is nonenumerable. There exists then a set $\alpha, \beta, \cdots, \tau, \cdots$ which has the limit zero and such that $F(\nu+\tau+m) \neq F(\nu+\tau+r), r, m=0, \pm 1, \pm 2, \cdots ; r \neq m$. Consider now the functions $y_{v+\tau+m}(x)$ and $P_{-\nu-\tau-p}(x)$ which are formed for $\lambda=\lambda_{m}{ }^{\prime}=F(\nu+\tau+m)$ and $\lambda=\lambda_{p}{ }^{\prime}=F(\nu+\tau+p)$ respectively. This is equivalent to replacing the $K$ of the boundary conditions by $K_{\tau}^{\prime}=e^{2 \pi i r} \cdot K$. These latter functions come under the previous case and we can write from (31)

We have

$$
\sum_{q=0}^{p-m} b_{-\nu-\tau-p, q} a_{\nu+\tau+m, p-m-q}=0 .
$$

$$
\underset{\tau \rightarrow 0}{\mathrm{~L}} b_{-\gamma-\tau-p, q}=b_{-\nu-p, q}, \underset{\tau \rightarrow 0}{\mathrm{~L}} a_{\nu+\tau+m, p-m-q}=a_{\nu+m, p-m-q} .
$$

This follows from the continuity of the function involved in the equations satisfied by the $a$ 's and $b$ 's. Since (31) is only a finite sum we may write

$$
\underset{\tau \rightarrow 0}{\mathrm{~L}} \sum_{q=0}^{p-m} b_{-\nu-\tau-p, q} a_{\nu+r+m, p-m-q}=\sum_{q=0}^{r-m} b_{-\nu-p q} a_{\nu+m, p-m-q}=0 .
$$

Comparing with the second equation of (32) we get

$$
\int P_{-\nu-p}(x) x^{-1} y_{\nu+m}(x) d x=0
$$

which proves the lemma.

The series (29) is a series of analytic functions of $x$ and $t$ and converges uniformly if $|x| \leqq R<|t|$; hence it represents an analytic function of $x$ and $t$. The difference

$$
(t-x)^{-1}-\sum_{m=0}^{\infty} P_{-\nu-m}(t) t^{-1} x^{-\nu} y_{\nu+m}(x)=\sum_{m=0}^{\infty} A_{m}(t) x^{m}
$$

is an analytic function of $x$ and has an ascending power series expansion in $x$. To determine the coefficients $A_{m}(t)$ let $t$ be any fixed point such that $0<|t|$ $<\Gamma$. Multiply through by $x^{-1} P_{-\nu-m}(x) d x$ and integrate along the contour 
$C_{1}$ defined by $|x|=R^{\prime \prime}<|t|<\Gamma$. This is legitimate since the series converges uniformly along this circle. From $(t-x)^{-1}$ we obtain $t^{-1} P_{-v-m}(t)$ and from the series we get also $t^{-1} P_{-\nu-m}(t)$ and hence the right hand side gives zero. We have for $m=0,1,2, \cdots$,

Now

$$
\sum_{r=0}^{\infty} \int_{C_{1}} A_{r}(t) x^{r-1} P_{-\nu-m}(x) d x=0 .
$$

$$
P_{-v-m}(x)=x^{-m}\left\{1+\sum_{s=1}^{m} b_{-y-m, s} x^{s}\right\} .
$$

Substituting in the above summation we see that if $r-m>0$ the integrals are zero, otherwise they are equal to $b_{-\nu-m, m-r} \cdot 2 \pi i$; that is,

$$
2 \pi i \sum_{r=0}^{m} A_{r}(t) b_{-\nu-m, m-r}=0 .
$$

The coefficient of $A_{m}(t)$ is $b_{-\nu-m, 0}$ or unity, and $A_{m}(t)$ is expressed as a linear combination of $A_{m-1}(t), A_{m-2}(t)$, etc. But for $m=0$ we have

$$
A_{0}(t) b_{-v-m, 0}=0 \text { or } A_{0}(t)=0 .
$$

Hence by induction $A_{m}(t)=0$ for every $m$. This proves

THEOREM III. Let $x$ and $t$ be two complex variables such that $|x| \leqq R^{\prime}<\Gamma$ and $\Gamma>|t| \geqq R>R^{\prime}$; then the expansion.

$$
(t-x)^{-1}=\sum_{m=0}^{\infty} t^{-1} P_{-v-m}(t) x^{-v} y_{v+m}(x)
$$

is valid. If $t$ is a variable point on the contour $C$ defined by the circle $|t|=R$ and $x$ a variable point within a smaller circle about $x=0$, then the expansion converges absolutely and uniformly with respect to $t$ and $x$.

5. Expansion of an arbitrary function. Since the expansion (33) converges uniformly with respect to $t$ on the circle $C$, then if $|x|<R$ we may write

$$
\begin{aligned}
f(x) & =\frac{1}{2 \pi i} \int_{C} \frac{f(t) d t}{t-x} \\
& =\sum_{m=0}^{\infty} x^{-v} y_{v+m}(x) \frac{1}{2 \pi i} \int_{C} f(t) P_{-v-m}(t) t^{-1} d t
\end{aligned}
$$


where $f(x)$ is analytic within and on the circle of radius $R$. We may state this as the following

THEOREM IV. Let $f(x)$ be a function of $x$ which is analytic and single-valued inside and upon the contour $C$ formed by the circle $|x|=R<\Gamma$; then the expansion

$$
x^{\prime} f(x)=\sum_{m=0}^{\infty} a_{m} y_{v+m}(x)
$$

where

$$
a_{m}=\frac{1}{2 \pi i} \int_{C} f(t) t^{-1} P_{-\nu-m}(t) d t,
$$

is valid in the circle $|x|=R^{\prime}$, where $R^{\prime}<R$. The expansion divided by $x^{\prime}$ converges absolutely and uniformly with respect to $x$ provided that $|x| \leqq R^{\prime}$.

The absolute and uniform convergence follows from bounding the coefficients $a_{m}$ and the function $x^{-v} y_{v+m}(x)$ by use of the corollary of $\S 3$.

Let the Taylor's series for $f(t)$ be

$$
f(t)=\sum_{s=0}^{\infty} \alpha_{s} t^{s}
$$

then

$$
\begin{aligned}
a_{m} & =\frac{1}{2 \pi i} \int_{C} f(t) t^{-1} P_{-,-m}(t) d t \\
& =\frac{1}{2 \pi i} \int_{C} t^{-1}\left\{\alpha_{0}+\alpha_{1} t+\cdots\right\}\left\{t^{-m}+b_{-\nu-m, 1} t^{-m-1}+\cdots+b_{-\nu-m, m}\right\} d t .
\end{aligned}
$$

The coefficient of $1 / t$ is $\sum_{n=0}^{m} b_{-\nu-m, m-n} \alpha_{n}$; hence

$$
a_{m}=\sum_{n=0}^{m} b_{-;-m, m-n} \alpha_{n} \quad(m=0,1, \cdots) .
$$

Let $f(x)$ be a single-valued analytic function in the annular region defined by $0<r \leqq|x| \leqq R^{\prime}<\Gamma$. Then by Cauchy's theorem

$$
f(x)=\frac{1}{2 \pi i} \int_{c} \frac{f(t) d t}{t-x}+\frac{1}{2 \pi i} \int_{c} \frac{f(t) d t}{x-t},
$$

where $C$ and $c$ are the contours $|t|=R^{\prime}<\Gamma$ and $0<|t|=r^{\prime}, r<R^{\prime}$, respectively. We use the expansion (33) for $1 /(t-x)$ in the first integral and (33) with $x$ and $t$ interchanged for the second integral. Taking into account that these expansions converge uniformly we evidently have 
THEOREM V. If $f(x)$ is analytic and single-valued in the annular region defined by

$$
0<r \leqq|x| \leqq R<\Gamma
$$

the expansion

$$
f(x)=\sum_{m=0}^{\infty} a_{m} x^{-\nu} y_{v+m}(x)+\sum_{m=0}^{\infty} a_{m}^{\prime} x^{-1} P_{-\nu-m}(x),
$$

where

$$
a_{m}=\frac{1}{2 \pi i} \int_{C} f(t) t^{-1} P_{-\nu-m}(t) d t, a_{m}^{\prime}=\int_{c} f(t) t^{-v} y_{v+m}(t) d t
$$

is valid and converges absolutely and uniformly with respect to $x$ when $r^{\prime} \leqq|x|$ $\leqq R^{\prime}$ where $r<r^{\prime}$ and $R^{\prime}<R$.

If the Laurent expansion of $f(x)$ is

$$
f(x)=\sum_{m=0}^{\infty} \alpha_{m} x^{-m}+\sum_{m=1}^{\infty} \alpha_{m}^{\prime} x^{-m}
$$

we can write

$$
a_{m}=\sum_{n=0}^{m} b_{-\nu-n, m-n} \alpha_{n}, \quad a_{m}^{\prime}=\sum_{n=0}^{\infty} a_{\nu+m+1, n} \alpha_{m}^{\prime}+n+1 .
$$

6. Extension to functions of several variables. Let $x_{1}, t_{1}, x_{2}, t_{2}, \cdots, x_{2}$, $t$. be complex variables and let $f\left(x_{1}, x_{2}, \cdots, x_{s}\right)$ be a single-valued analytic function when $\left|x_{1}\right|<R_{1} \leqq \Gamma,\left|x_{2}\right|<R_{2} \leqq \Gamma, \cdots,\left|x_{8}\right|<R_{\mathrm{s}} \leqq \Gamma$. The expansion (33) for $\left(t_{k}-x_{k}\right)^{-1}$ converges absolutely and uniformly with respect to $x_{k}$ if $t_{k}$ is restricted to the circle $\left|t_{k}\right|=R_{k}^{\prime}<R_{k}$ and $\left|x_{k}\right|<R_{k}^{\prime}$. A product of such absolutely convergent expansions for $k=1,2, \cdots, s$ will have the corresponding property for $x_{1}, \cdots, x_{s}$ and $t_{1}, \cdots, t_{s}$. We also have

$$
f\left(x_{1}, x_{2}, \cdots, x_{8}\right)=\left(\frac{1}{2 \pi i}\right)^{8} \int_{C_{1}} \int_{C_{2}} \cdots \int_{C_{8}} \frac{f\left(t_{1}, \cdots, t_{8}\right) d t_{8} \cdots d t_{1}}{\left(t_{1}-x_{1}\right) \cdots\left(t_{8}-x_{8}\right)},
$$

where $C_{1}, C_{2}, \cdots, C_{8}$ are contours defined by the circles

$$
\left|t_{1}\right|=R_{1}^{\prime},\left|t_{2}\right|=R_{2}^{\prime}, \cdots,\left|t_{s}\right|=R_{s}^{\prime} .
$$

The following extension is evident. 
TheOREM VI. Let $f\left(x_{1}, x_{2}, \cdots, x_{8}\right)$ be a function of $x_{1}, x_{2}, \cdots, x_{8}$ which is single-valued and analytic when $x_{1}, x_{2}, \cdots, x_{2}$ respectively satisfy the conditions $\left|x_{1}\right|<R_{1},\left|x_{2}\right|<R_{2}, \cdots,\left|x_{8}\right|<R_{8}$; then the expansion

$$
\begin{aligned}
& f\left(x_{1}, x_{2}, \cdots, x_{s}\right)=\sum_{m_{1}=0}^{\infty} \sum_{m_{2}=0}^{\infty} \cdots
\end{aligned}
$$

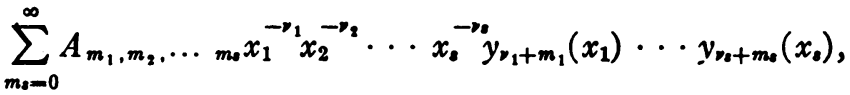

where

$$
\begin{aligned}
& A_{m_{1}, \cdots, m_{\mathrm{s}}}=\left(\frac{1}{2 \pi i}\right)^{\prime} \int_{C_{1}} \cdots \\
& \int_{c_{s}}\left(t_{1} t_{2} \cdots t_{s}\right)^{-1} f\left(t_{1}, \cdots, t_{s}\right) P_{-v_{1}-m_{1}}\left(t_{1}\right) \cdots P_{-y_{s}-m_{0}}\left(t_{s}\right) d t_{s} \cdots d t_{1},
\end{aligned}
$$

is valid and converges absolutely and uniformly when $x_{1}, \cdots, x_{s}$ are respectively inside the circles $\left|x_{1}\right|=r_{1}, \cdots,\left|x_{8}\right|=r_{s}$, where $r_{1}<R_{1}, \cdots, r_{8}<R_{8}$.

It is to be noted that it is not necessary that the expansions of the various $\left(t_{k}-x_{k}\right)$ used in proving the above theorem arise out of a single differential system. The various $y_{v_{k}+m_{k}}\left(x_{k}\right)$ may be replaced by a solution of a different differential system of the type considered here.

UNIVERSITY OF ILLINOIS, Urbana, ILL. 